\title{
Exotic neutrinoless double beta decay with Majoron-like emission
}

Cite as: AIP Conference Proceedings 2165, 020006 (2019); https://doi.org/10.1063/1.5130967

Published Online: 25 October 2019

Frank F. Deppisch

ARTICLES YOU MAY BE INTERESTED IN

Experimental studies of axial-vector weak couplings for double beta decays by nuclear and muon CERs

AIP Conference Proceedings 2165, 020007 (2019); https://doi.org/10.1063/1.5130968

Lepton flavor violation processes in nuclei

AIP Conference Proceedings 2165, 020004 (2019); https://doi.org/10.1063/1.5130965

Neutrinoless double beta decay of atomic nuclei

AIP Conference Proceedings 2165, 020012 (2019); https://doi.org/10.1063/1.5130973

Lock-in Amplifiers

... and more, from DC to $600 \mathrm{MHz}$

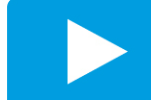

Watch 


\title{
Exotic Neutrinoless Double Beta Decay with Majoron-like Emission
}

\author{
Frank F. Deppisch ${ }^{\text {a) }}$ \\ Department of Physics and Astronomy, University College London, \\ Gower Street, London WC1E 6BT, United Kingdom. \\ a)Corresponding author: f.deppisch@ucl.ac.uk
}

\begin{abstract}
We discuss a novel mode of neutrinoless double- $\beta$ decay with emission of a light Majoron-like scalar particle $\phi$ which couples via an effective 7-dimensional operator with a $(V+A)$ lepton current and $(V \pm A)$ quark currents leading to a long-range contribution. Future double- $\beta$ decay searches are sensitive to scales of the order $\Lambda_{\mathrm{NP}} \approx 1 \mathrm{TeV}$ for the effective operator. In a left-right symmetric model, this mode can probe right-handed $W$ boson masses up to $25 \mathrm{TeV}$.
\end{abstract}

\section{DOUBLE BETA DECAYS}

Double $\beta$ decays are sensitive probes of physics beyond the Standard Model (SM). Two neutrino double $\beta$ ( $2 v \beta \beta$ ) decay, allowed in the SM, is the rarest process ever observed with half lives of the order $T_{1 / 2} \sim 10^{21} \mathrm{y}$. Neutrinoless double $\beta(0 v \beta \beta)$ decay, with no observation of any missing energy, is clearly the most important mode beyond the $\mathrm{SM}$ as it probes the Majorana nature and mass $m_{v}$ of light neutrinos, with current experiments having a sensitivity of $T_{1 / 2} \sim\left(0.1 \mathrm{eV} / m_{v}\right)^{2} \times 10^{26} \mathrm{y}$. More generally, it is a crucial test for any new physics scenario that violates lepton number by two units [1].

In addition, one or more exotic neutral particles may also be emitted, with a signature of anomalous missing energy beyond that expected in $2 v \beta \beta$ decay. An often studied set of theories involve the emission of one or two so called Majorons $J$. The first such proposed Majoron was a Goldstone boson associated with the spontaneous breaking of lepton number symmetry [2,3], coupling to a neutrino $v$ as $g_{J} v v J$, see Fig. 1 (left). Current searches have a sensitivity of the order $T \sim\left(10^{-5} / g_{J}\right)^{2} \times 10^{24} \mathrm{y}$. The term Majoron has been used in a wider sense, simply referring to a neutral scalar or vector particle [4]. Though originally massless, it may also be a massive but light particle [5, 6, 7] that can potentially play the role of Dark Matter $[8,9,10]$. Searches for extra particles in double $\beta$ decay are crucial in understanding neutrinos. Most importantly, violation of lepton number by two units and thus the Majorana nature of neutrinos can only be firmly established in the case of $0 v \beta \beta$ decay.

Not all possible emission modes have been discussed in the literature. Existing experimental searches so far focus on the emission of one or two Majorons originating from the intermediate neutrino exchanged in the process. The different Majoron scenarios have been classified into several categories, all of which assume SM $(V-A)$ charged currents with the electrons and quarks. In Ref. [11], we instead considered $0 v \beta \beta \phi$ decay with emission of a light neutral scalar $\phi$ from a single effective dimension-7 operator of the form $\Lambda_{\mathrm{NP}}^{-3}(\bar{u} \mathscr{O} d)(\bar{e} \mathscr{O} v) \phi$, see Fig. 1 (center), with the fermion currents having a different chiral structure from that in the SM.

\section{RIGHT-HANDED CURRENTS ACCOMPANIED BY A SCALAR}

We consider processes where right- and left-handed electrons are emitted along with a scalar $\phi$ considering $(V+A)$ and $(V-A)$ currents,

$$
\mathscr{L}_{0 v \beta \beta \phi}=\frac{G_{F} \cos \theta_{C}}{\sqrt{2}}\left(j_{L}^{\mu} J_{L \mu}+\frac{\varepsilon_{R L}^{\phi}}{m_{p}} j_{R}^{\mu} J_{L \mu} \phi+\frac{\varepsilon_{R R}^{\phi}}{m_{p}} j_{R}^{\mu} J_{R \mu} \phi\right)+\text { h.c.. }
$$

Here, the Fermi constant is denoted by $G_{F}$, the Cabbibo angle by $\theta_{C}$, and the leptonic and hadronic currents are $j_{L, R}^{\mu}=\bar{e} \gamma^{\mu}\left(1 \mp \gamma_{5}\right) v$ and $J_{L, R}^{\mu}=\bar{u} \gamma^{\mu}\left(1 \mp \gamma_{5}\right) d$, respectively. The 4-spinor field of the light electron neutrino is denoted by $v$, either defined by $v=v_{L}+v_{L}^{c}$ (i.e. a Majorana spinor constructed from the SM active left-handed neutrino $v_{L}$ ) or $v=v_{L}+v_{R}$ (a Dirac spinor constructed from the SM $v_{L}$ and a new SM-sterile right-handed neutrino $v_{R}$ ). Whether the light neutrinos are of Majorana or Dirac type and whether total lepton number is broken or conserved is of crucial importance for an underlying model (determined by the chosen lepton numbers for $v_{R}$ and $\phi$ ) but as far as the effective

Workshop on Calculation of Double-beta-decay Matrix Elements (MEDEX'19)

AIP Conf. Proc. 2165, 020006-1-020006-4; https://doi.org/10.1063/1.5130967

Published by AIP Publishing. 978-0-7354-1910-0/\$30.00 

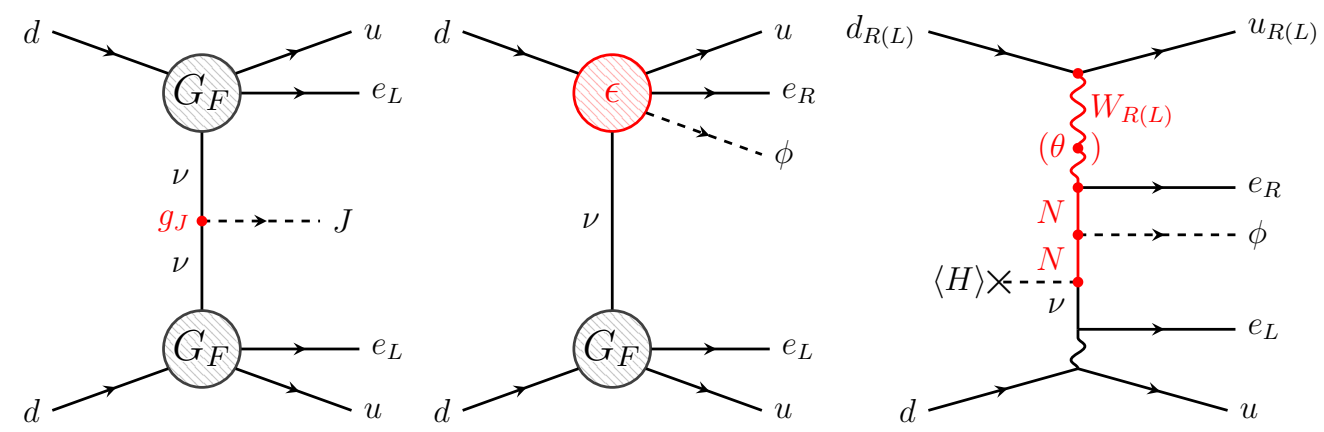

FIGURE 1. Diagrams for ordinary $0 v \beta \beta J$ Majoron decay (left), $0 v \beta \beta \phi$ decay triggered by an effective operator of the form $\Lambda_{\mathrm{NP}}^{-3}(\bar{u} \mathscr{O} d)(\bar{e} \mathscr{O} v) \phi$ (center) and a possible realization of the latter in a Left-Right symmetric model (right).

interactions in Eq. (1) are concerned, this does not play a role in our calculations. The proton mass $m_{p}$ is introduced in the exotic interactions as normalization to make the effective coupling constants $\varepsilon_{R L}^{\phi}$ and $\varepsilon_{R R}^{\phi}$ dimensionless, in analogy to the effective operator treatment of $0 v \beta \beta$ decay [1,12]. The above Lagrangian will give rise to processes depicted in Fig. 1 (center), where the SM $(V-A)$ Fermi interaction, the first term in Eq. (1), meets one of the exotic operators. In this case, the momentum part in the numerator of the neutrino propagator contributes, rather than the mass. Throughout, we consider the first generation electron and neutrino only.

\section{DECAY RATE AND DISTRIBUTIONS}

Summing over all intermediate nuclear states $N$, the amplitude of $0_{I}^{+} \rightarrow 0_{F}^{+} 0 v \beta \beta \phi$ decay can be written as [11]

$$
\begin{aligned}
\mathscr{M} & =\varepsilon_{R X}^{\phi} \frac{\left(G_{F} \cos \theta_{C}\right)^{2}}{\sqrt{2} m_{p}} \sum_{N} \int d^{3} x d^{3} y \int \frac{d^{3} q}{2 \pi^{2} \omega} \phi(\mathbf{y}) e^{i \mathbf{q}(\mathbf{x}-\mathbf{y})} \\
& \times\left\{\left[\frac{J_{L X}^{\rho \sigma}(\mathbf{x}, \mathbf{y}) u_{\rho \sigma}^{L}\left(E_{1} \mathbf{x}, E_{2} \mathbf{y}\right)}{\omega+\mu_{N}-\frac{1}{2}\left(E_{1}-E_{2}-E_{\phi}\right)}-\frac{J_{X L}^{\rho \sigma}(\mathbf{x}, \mathbf{y}) u_{\rho \sigma}^{R}\left(E_{1} \mathbf{x}, E_{2} \mathbf{y}\right)}{\omega+\mu_{N}-\frac{1}{2}\left(E_{1}-E_{2}+E_{\phi}\right)}\right]-\left[E_{1} \leftrightarrow E_{2}\right]\right\} .
\end{aligned}
$$

Here, $X=L, R$ correspond to $\varepsilon_{R L}^{\phi}, \varepsilon_{R R}^{\phi}$ and $\mu_{N}=E_{N}-E_{I}+Q_{\beta \beta} / 2+m_{e}$ with $E_{I}$ and $E_{N}$ the energies of the initial and intermediate nucleus, respectively. The energies of the two outgoing electrons and the Majoron are $E_{1,2}$ and $E_{\phi}$, respectively, and the available kinetic energy release is $Q_{\beta \beta}$. The nucleon and lepton currents are denoted by $J_{Y X}^{\rho \sigma}(\mathbf{x}, \mathbf{y})$ $(X, Y=L, R)$ and $u_{\rho \sigma}^{L, R}\left(E_{1} \mathbf{x}, E_{2} \mathbf{y}\right)$, respectively. The internal neutrino propagates between the interaction points $\mathbf{x}$ and $\mathbf{y}$ with momentum $q^{\mu}=(\omega, \mathbf{q})$. The above amplitude is antisymmetric under the exchange of the two electrons.

In Eq. (2), the Majoron energy $E_{\phi}$ is added or subtracted depending on whether the electron labelled 1 or 2 is being emitted from the exotic operator. The Majoron makes a crucial difference, as $E_{\phi}$ goes together with $\left(E_{1}-E_{2}\right)$ and not with the term proportional to the intermediate nuclei energy $\mu_{N}$ as for an ordinary Majoron. A dependence on $E_{\phi}$ will thus appear through the matrix element in addition to that through the phase space. The differential rate for the decay, along with various distributions and the total rate can then be calculated using the formalism of Ref. [13], where we assumed that the exotic $\phi$ Majoron is massless in our calculations.

The crucial distribution is with respect to the sum of the kinetic energies of the detected electrons. With the SM $2 v \beta \beta$ decay as irreducible background to any exotic signal, it is important to calculate it precisely. In Fig. 2 (left), we compare the normalized total electron kinetic energy distribution of $0 v \beta \beta \phi$ decay with that of $2 v \beta \beta$ decay and ordinary $0 v \beta \beta J$ Majoron decay (with spectral index $n=1$ ) for the isotope ${ }^{136} \mathrm{Xe}$. The distribution associated with $\varepsilon_{R L}^{\phi}$ is very similar to ordinary $0 v \beta \beta J$ decay, while the introduction of a hadronic right-handed current in the $\varepsilon_{R R}^{\phi}$ term changes considerably the shape of the distribution. In both cases, the spectral index still corresponds to $n=1$ with the characteristic onset near the kinematic endpoint. We emphasize that because of the different shape, a dedicated signal over background analysis is required to determine the experimental sensitivity on the effective parameters $\varepsilon_{R L}^{\phi}$ and $\varepsilon_{R R}^{\phi}$ precisely. 

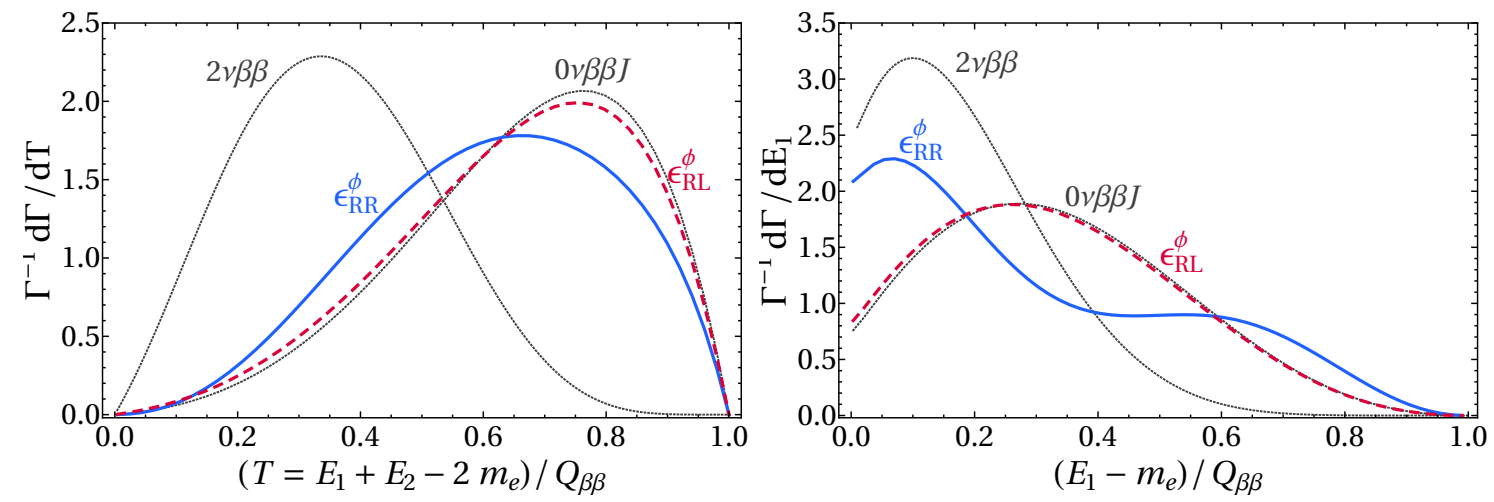

FIGURE 2. Left: Normalized $0 v \beta \beta \phi$ decay distributions in the total kinetic energy of the electrons for ${ }^{136} \mathrm{Xe}$. Right: Normalized $0 v \beta \beta \phi$ decay distribution in the single electron kinetic energy distribution for ${ }^{82} \mathrm{Se}$. The blue solid and red dashed lines correspond to the $\varepsilon_{R R}^{\phi}$ and $\varepsilon_{R L}^{\phi}$ cases, respectively. The corresponding distributions for the SM $2 v \beta \beta$ decay and ordinary $0 v \beta \beta J$ Majoron decay (spectral index $n=1$ ) are given for comparison.

Experiments like NEMO-3 and SuperNEMO are able to measure the individual electron energies. In right-handed current scenarios without emission of a Majoron, the single energy distribution exhibits a distinctive valley-type shape. This occurs as the dominant term is proportional to $\left(E_{1}-E_{2}\right)$ for the corresponding $\varepsilon_{R R}$ term, as a result of the antisymmetry with respect to electron exchange. In our scenario, depicted in Fig. 2 (right), part of the energy is being carried away by the Majoron, shifting the distribution towards lower electron energies and softening the characteristic valley-type distribution for $\varepsilon_{R R}^{\phi}$. The distribution does not vanish for $E_{1}-m_{e}=\frac{1}{2} Q_{\beta \beta}$, but is still significantly different from that of ordinary Majoron emission.

Angular correlations can also be used to distinguish between left- and right-handed currents $[14,15]$. Integrating over the electron energies one obtains the purely angular correlation $d \Gamma / d \cos \theta=\Gamma / 2(1+k \cos \theta)$, with the total decay rate $\Gamma$. The coefficient $k$ is $k_{R L}^{\phi}=+0.70$ (electrons are dominantly emitted collinearly) and $k_{R R}^{\phi}=-0.05$ (electrons are emitted nearly isotropically) in our $0 v \beta \beta \phi$ scenarios $\left({ }^{82} \mathrm{Se}\right)$. For comparison, the angular correlation factor for SM $2 v \beta \beta$ decay is $k^{2 v \beta \beta}=-0.66$ and $k^{J}=-0.80$ for ordinary Majoron emission; i.e., the electrons are dominantly emitted back-to-back.

The sensitivity of existing and planned future double $\beta$ decay searches on the effective couplings $\varepsilon_{R L}^{\phi}$ and $\varepsilon_{R R}^{\phi}$ of $0 v \beta \beta \phi$ decay is estimated to be of the order of a few $10^{-2}$ to $10^{-4}$ and $10^{-3}$ to $10^{-5}$, respectively [11]. These estimates are based on an extrapolation of existing Majoron search analysis but as the signal in our scenarios can be considerably different, dedicated analyses are required. The future sensitivities on the effective couplings $\varepsilon_{R L}^{\phi}$ and $\varepsilon_{R R}^{\phi}$ may be translated into effective operator scales $\Lambda_{\mathrm{NP}} \approx 270 \mathrm{GeV}$ to $1.3 \mathrm{TeV}$, respectively. As noted before, we assume a massless $\phi$ in deriving these limits; they remain essentially unchanged for masses small compared to $Q_{\beta \beta}$, $m_{\phi} \lesssim 0.2 \mathrm{MeV}$ and are of the same order for $m_{\phi} \lesssim 1 \mathrm{MeV}$, but will deteriorate as $m_{\phi} \rightarrow Q_{\beta \beta}$ (for a recent analysis in ordinary Majoron emission, see Ref. [10]). Constraints on our operators may also be set from other processes, such as exotic decay modes of the pion, $\pi^{-} \rightarrow e^{-} \bar{v}_{e} \phi$. As we consider only $V+A$ currents, helicity suppression will still apply and the limits are expected to be correspondingly weak, we roughly estimate $\Lambda_{\mathrm{NP}} \gtrsim 15 \mathrm{GeV}$.

\section{MODEL EXAMPLE}

An ultraviolet scenario generating the effective operators in Eq. (1) is suggested in Left-Right symmetric models $[16,17,18]$ where the SM $W$ and $v$ are replaced by their right-handed counterparts $W_{R}$ and $N$. The heavy neutrino $N$ then mixes with $v$ via a Yukawa coupling $y_{v}$ once the SM Higgs boson acquires its vacuum expectation value $\langle H\rangle=174 \mathrm{GeV}$. A massless or light scalar $\phi$ is not part of the minimal Left-Right symmetric model which thus needs to be modified, e.g. by keeping the $U(1)_{B-L}$ symmetry global or by extending its scalar sector. Charging $\phi$ under lepton number allows coupling to $N$ with a strength $y_{N}$. The corresponding diagram is shown in Fig. 1 (right). We can 
then identify $G_{F} \cos \theta_{C} /\left(\sqrt{2} m_{p}\right) \varepsilon_{R L}^{\phi}=g_{R} g_{L} \theta y_{N} y_{v}\langle H\rangle \cos \theta_{C} /\left(8 m_{W}^{2} m_{N}^{2}\right)$, resulting in the estimate

$$
\frac{T_{1 / 2}^{\mathrm{Xe}}}{10^{25} \mathrm{y}} \approx\left(\frac{1.4 \times 10^{-4}}{g_{R}^{2} \kappa y_{N} y_{v}}\right)^{2}\left(\frac{m_{W_{R}}}{25 \mathrm{TeV}}\right)^{4}\left(\frac{m_{N}}{100 \mathrm{MeV}}\right)^{4} .
$$

Here, $g_{R}$ is the gauge coupling constant and $\theta_{C}^{R}$ the equivalent of the Cabbibo angle, both associated with the $S U(2)_{R}$ of the Left-Right symmetric model. The above equivalence is triggered by the $W_{R}-W$ mixing $\theta$. Its value is generically expected to be $\theta=\kappa g_{R} m_{W}^{2} /\left(g_{L} m_{W_{R}}^{2}\right)$ where $\kappa=\mathscr{O}(1)$. A more detailed description of the Left-Right symmetric model is given in Ref. [11], in which ordinary $0 v \beta \beta$ decay is not allowed and only our $0 v \beta \beta \phi$ mode would occur.

The interactions in Eq. (1) could be extended in several directions. Most straightforwardly, one can generalize Eq. (1) by including scalar and tensor fermion currents to incorporate all possible Lorentz-invariant combinations. The Majoron may also couple derivatively, if originating as a Goldstone boson; this would increase the number of possible Lorentz-invariant combinations. Alternatively, if the exotic particle is a vector boson $a^{\mu}$ [4], such as a dark photon, the fermion currents can couple to it via the vector field itself as well as its field strength tensor $f^{\mu v}$. An even number of exotic neutral fermions $\chi$ may also be emitted but this would quickly increase the dimension of the corresponding effective operator. Instead, they may also originate from the internal neutrino via a dimension- 6 operator of the form $\Lambda_{\mathrm{NP}}^{-2} v v \chi \chi$ [19]. Exploring such alternatives to the well-studied neutrinoless double $\beta$ decay is imperative in order to be able to draw reliable conclusions on the nature of neutrino mass generation.

\section{ACKNOWLEDGMENTS}

The speaker and author of this proceeding contribution gratefully acknowledges the discussion and cooperation of his co-authors of Ref. [11], Ricardo Cepedello, Lorena González, Chandan Hati and Martin Hirsch, on which this report is based.

\section{REFERENCES}

1. F. F. Deppisch, M. Hirsch, and H. Päs, “Neutrinoless Double Beta Decay and Physics Beyond the Standard Model," J. Phys. G39, 124007 (2012), arXiv:1208.0727 [hep-ph].

2. Y. Chikashige, R. N. Mohapatra, and R. D. Peccei, “Are There Real Goldstone Bosons Associated with Broken Lepton Number?" Phys. Lett. 98B, 265-268 (1981).

3. G. B. Gelmini and M. Roncadelli, "Left-Handed Neutrino Mass Scale and Spontaneously Broken Lepton Number," Phys. Lett. 99B, 411-415 (1981).

4. C. D. Carone, "Double beta decay with vector majorons," Phys. Lett. B308, 85-88 (1993), arXiv:hep-ph/9302290 [hep-ph].

5. P. Bamert, C. P. Burgess, and R. N. Mohapatra, "Multi - Majoron modes for neutrinoless double beta decay," Nucl. Phys. B449, 25-48 (1995), arXiv:hep-ph/9412365 [hep-ph].

6. M. Hirsch, H. V. Klapdor-Kleingrothaus, S. G. Kovalenko, and H. Päs, "On the observability of majoron emitting double beta decays," Phys. Lett. B372, 8-14 (1996), [,933(1995)], arXiv:hep-ph/9511227 [hep-ph].

7. K. Blum, Y. Nir, and M. Shavit, "Neutrinoless double-beta decay with massive scalar emission," Phys. Lett. B785, 354-361 (2018), arXiv: 1802.08019 [hep-ph].

8. V. Berezinsky and J. W. F. Valle, “The KeV majoron as a dark matter particle,” Phys. Lett. B318, 360-366 (1993), arXiv:hep-ph/9309214 [hep-ph].

9. C. Garcia-Cely and J. Heeck, "Neutrino Lines from Majoron Dark Matter," JHEP 05, 102 (2017), arXiv:1701.07209 [hep-ph].

10. T. Brune and H. Päs, "Majoron Dark Matter and Constraints on the Majoron-Neutrino Coupling," (2018), arXiv:1808.08158 [hep-ph].

11. R. Cepedello, F. F. Deppisch, L. González, C. Hati, and M. Hirsch, "Neutrinoless Double- $\beta$ Decay with Nonstandard Majoron Emission," Phys. Rev. Lett. 122, 181801 (2019), arXiv:1811.00031 [hep-ph].

12. H. Päs, M. Hirsch, H. V. Klapdor-Kleingrothaus, and S. G. Kovalenko, "Towards a superformula for neutrinoless double beta decay," Phys. Lett. B453, 194-198 (1999).

13. M. Doi, T. Kotani, and E. Takasugi, "Double beta Decay and Majorana Neutrino," Prog. Theor. Phys. Suppl. 83, 1 (1985).

14. M. Doi, T. Kotani, H. Nishiura, and E. Takasugi, "The Energy Spectrum and the Angular Correlation in the Beta Beta Decay," Prog. Theor. Phys. 70, 1353 (1983).

15. R. Arnold et al. (SuperNEMO), "Probing New Physics Models of Neutrinoless Double Beta Decay with SuperNEMO," Eur. Phys. J. C70, 927-943 (2010), arXiv:1005.1241 [hep-ex].

16. J. C. Pati and A. Salam, "Lepton Number as the Fourth Color," Phys. Rev. D10, 275-289 (1974), [Erratum: Phys. Rev.D11,703(1975)].

17. R. N. Mohapatra and J. C. Pati, "Left-Right Gauge Symmetry and an Isoconjugate Model of CP Violation," Phys. Rev. D11, 566-571 (1975).

18. R. N. Mohapatra and J. C. Pati, “A Natural Left-Right Symmetry,” Phys. Rev. D11, 2558 (1975).

19. W.-C. Huang and F. F. Deppisch, "Dark matter origins of neutrino masses," Phys. Rev. D91, 093011 (2015), arXiv:1412.2027 [hep-ph]. 